\title{
Der DEGUM-Vorstand im Interview - PD Dr. med Kai-Sven Heling
}

Kai-Sven Heling ist künftiger Präsident der DEGUM (2016 bis 2018) und leitet eine Praxis für Pränatalmedizin in Berlin. Seit 2 Jahren ist er DEGUM-Vorstandsmitglied und setzt sich seither insbesondere für die Ultraschall-Ausbildung ein. Einen besonderen Fokus legt er auf die berufspolitische Positionierung der DEGUM.

? Wir holen Sie ganz am Anfang ab: Wann haben Sie sich für die DEGUM entschieden und was hat Sie dazu motiviert?

Meine berufliche Laufbahn begann mit dem Ultraschall in der Pränataldiagnostik der Charité. Es war daher naheliegend, Mitglied der DEGUM zu werden, 1996 bin ich dann der Fachgesellschaft beigetreten. Das Konzept der interdisziplinären Gesellschaft hatte mich überzeugt, und meine ersten wissenschaftlichen Vorträge habe ich auch dort gehalten.

? Welche persönlichen Ideen / Visionen haben Sie in das Vorstandsamt gebracht?

Ich bin der persönlichen Überzeugung, dass man auch Verantwortung übernehmen muss. Da der Ultraschall den Hauptteil meiner täglichen und auch wissenschaftlichen Arbeit ausmacht, möchte ich dies aktiv gestalten. Hier liegt mir insbesondere die Ausbildung am Herzen.
? Wie haben Sie ihre Amtszeit erlebt, welches Fazit ziehen sie daraus?

Die Zusammenarbeit im Vorstand hat sich als sehr harmonisch und konstruktiv erwiesen. Man hat immer den Eindruck, dass alle am gleichen Strang ziehen und die Interessen der Fachgesellschaft oben angestellt werden. Es ist wichtig, und ein Verdienst des derzeitigen Präsidenten, Dirk Becker, eine solche Atmosphäre geschaffen zu haben.

? Worin sehen Sie aktuell die größten gesundheitspolitischen Herausforderungen für die DEGUM?

Ich denke, die DEGUM wird sich künftig nicht mehr nur wissenschaftlich, sondern auch berufspolitisch positionieren müssen. Dies ist insbesondere deshalb wichtig, damit wir weiterhin den Stellenwert des Ultraschalls in der Medizin gut abbilden können.
? Wie schätzen Sie die zukünftige Bedeutung des Ultraschalls in der Medizin ein?

Enorm wichtig! Es ist eine Methode, die immer verfügbar ist und die Bildauflösung nimmt weiter zu. Auf der anderen Seite ist die Ausbildung mitunter nicht so, wie wir es uns wünschen: Der Untersucher selbst diskreditiert mitunter den Ultraschall gegenüber anderen bildgebenden Verfahren wie MRT und ähnlichen Methoden. Das Potenzial des Ultraschalls wird daher in der Praxis meist weiterhin unterschätzt. Die Wunderwelt des Lebens im Uterus, dargestellt durch mittlerweile hochsensible Ultraschallgeräte, fasziniert mich jeden Tag neu.

? Mit welchen Themen beschäftigen Sie sich derzeit im klinischen Alltag/der klinischen Forschung?

Mich interessieren insbesondere die frühe fetale Echokardiografie, die fetale Neurosonografie, die fetale Lunge, die 3D-Sonografie und die Prognoseeinschätzung von Fehlbildungen.

? Wann hatten Sie zum 1. Mal einen Schallkopf in der Hand? Was hat sich seitdem verändert?

Ich hielt das 1. Mal 1989 als Student im Rahmen meiner damaligen Diplomarbeit den Schallkopf in der Hand. Die Bedienbarkeit der Geräte ist deutlich einfacher geworden. Die Bilder sind selbsterklärend. 